\title{
Computed tomography quantification of emphysema in people living with HIV and uninfected controls
}

\author{
Andreas Ronit (1) ${ }^{1}$, Thomas Kristensen², Vilde S. Hoseth ${ }^{1}$, Dalia Abou-Kassem¹, \\ Jørgen T. Kühl ${ }^{3}$, Thomas Benfield $\mathbb{1}^{4}$, Jan Gerstoft ${ }^{1}$, Shoaib Afzal ${ }^{5}$, \\ Børge G. Nordestgaard ${ }^{5,6}$, Jens D. Lundgren ${ }^{7}$, Jørgen Vestbo ${ }^{8}$, \\ Klaus F. Kofoed ${ }^{2,3}$ and Susanne D. Nielsen ${ }^{1}$ on behalf of the Copenhagen \\ Comorbidity in HIV Infection (COCOMO) study group
}

\begin{abstract}
Affiliations: 'Viro-immunology Research Unit, Dept of Infectious Diseases 8632, Rigshospitalet, University of Copenhagen, Copenhagen, Denmark. ${ }^{2}$ Dept of Radiology, Rigshospitalet, University of Copenhagen, Copenhagen, Denmark. ${ }^{3}$ Dept of Cardiology, Rigshospitalet, University of Copenhagen, Copenhagen, Denmark. ${ }^{4}$ Dept of Infectious Diseases, Hvidovre Hospital, University of Copenhagen, Hvidovre, Denmark. ${ }^{5}$ The Copenhagen General Population Study, Dept of Clinical Biochemistry, Herlev and Gentofte Hospital, Copenhagen University Hospital, Herlev, Denmark. ${ }^{6}$ Faculty of Health and Medical Sciences, University of Copenhagen, Copenhagen, Denmark. ${ }^{7} \mathrm{CHIP}$, Dept of Infectious Diseases 8632, Rigshospitalet, University of Copenhagen, Copenhagen, Denmark. ${ }^{8}$ Division of Infection, Immunity and Respiratory Medicine, University of Manchester, Manchester, UK.
\end{abstract}

Correspondence: Susanne D. Nielsen, Viro-immunology Research Unit, Dept of Infectious Diseases 8632, Copenhagen University Hospital, Blegdamsvej 9B, DK-2100 Copenhagen, Denmark.

E-mail: sdnadadlnet.dk

@ERSpublications

Well-treated HIV infection is not associated with emphysema http://ow.ly/2IA430khHvy

Cite this article as: Ronit A, Kristensen T, Hoseth VS, et al. Computed tomography quantification of emphysema in people living with HIV and uninfected controls. Eur Respir J 2018; 52: 1800296 [https://doi. org/10.1183/13993003.00296-2018].

ABSTRACT People living with HIV (PLWH) may be more susceptible to the development of emphysema than uninfected individuals. We assessed prevalence and risk factors for emphysema in PLWH and uninfected controls. Spirometry and chest computed tomography scans were obtained in PLWH from the Copenhagen Comorbidity in HIV Infection (COCOMO) study and in uninfected controls from the Copenhagen General Population Study (CGPS) who were $>40$ years. Emphysema was quantified using a low attenuation area $<-950$ Hounsfield units (\%LAA-950) and the 15th percentile density index (PD15) and assessed by semi-quantitative visual scales. Of 742 PLWH, 21.2\% and $4.7 \%$ had emphysema according to the \%LAA-950 threshold with cut-offs at $5 \%$ and $10 \%$, respectively. Of 470 uninfected controls, these numbers were $24.3 \%(\mathrm{p}=0.23)$ and $4.0 \%(\mathrm{p}=0.68)$. HIV was not associated with emphysema (adjusted OR 1.25, 95\% CI $0.68-2.36$ for \%LAA-950 >10\%) by PD15 or by visually assessed emphysema. We found no interaction between HIV and cumulative smoking. Breathlessness and sputum production were more common in PLWH with emphysema, and emphysema seemed to be more prevalent in PLWH with airflow limitation. HIV was therefore not independently associated with emphysema, but the clinical impact of emphysema was greater in PLWH than in uninfected controls. 


\section{Introduction}

AIDS-related emphysema was first reported in a case series in the late 1980s [1]. Several smaller studies have subsequently suggested that people living with HIV (PLWH) may develop emphysema at an earlier age and independently of tobacco smoking [2-4].

Specific disease mechanisms potentially contributing to the destruction of lung parenchyma in PLWH have been recognised, including accumulation of cytotoxic T-cells in the lungs in the absence of combination antiretroviral therapy (cART) $[3,5]$, and increased oxidant stress in the absence of cART [6, 7]. Even during cART, alveolar macrophages may act as long-lived reservoirs of HIV [8,9]. They can induce local inflammation and immune activation and produce proteases $[9,10]$. Moreover, because emphysema originates around the small airways, it is noteworthy that HIV binds to human airway progenitor cells [11] and that never-smoking PLWH have evidence of small airway dysfunction [12]. However, while the understanding of emphysema pathogenesis in PLWH has increased, the majority of observational computed tomography (CT) studies in the cART era have not included uninfected controls $[3,13-15]$, have been restricted to rather small samples [2-4] or have not included quantitative measures of emphysema $[3,4,16]$. Thus, whether HIV infection per se is associated with emphysema or emphysema severity in the current cART era still needs to be determined.

To address this question we performed CT densitometry and visual evaluation of emphysema in a population of PLWH and uninfected controls. A large number of participants enabled us to evaluate emphysema according to different thresholds of the percentage of low attenuation area (\%LAA) and to perform interaction analyses in order to determine whether the effects of age and smoking are augmented by HIV. Finally, we assessed whether an emphysematous phenotype is more common in PLWH with airflow limitation, and whether the clinical significance of emphysema differs according to HIV status.

\section{Methods}

Study design, study subjects and ethics

The Copenhagen Comorbidity in HIV Infection (COCOMO) study has been described elsewhere [17, 18]. In brief, the COCOMO study is a prospective study evaluating prevalence, incidence and pathogenesis of non-AIDS comorbidity in PLWH. Chest CT imaging was performed from February 2015 to April 2016 for PLWH and from November 2015 to October 2016 for uninfected controls. Uninfected controls were recruited from the Copenhagen General Population Study (CGPS) [19, 20]. Only individuals >40 years underwent CT imaging in the CGPS. Thus, in order to obtain comparable samples from the two cohorts, we only included COCOMO participants $>40$ years for the present study. Because women are overrepresented in the CGPS compared to the COCOMO study, we included every fifth woman from the CGPS to obtain balanced samples. Moreover, because individuals in the CGPS tend to be older, we only included every fifth individual $>70$ years. A flow diagram summarising inclusion has been included as supplementary material. Ethical approval was obtained from the Regional Ethics Committee of Copenhagen (H-15017350; H-KF-01-144/01). Written informed consent was obtained from all participants.

\section{Data collection}

Information relating to respiratory risk factors and self-reported respiratory morbidity was obtained through identical questionnaires used in the COCOMO study and the CGPS. Breathlessness was defined by the modified Medical Research Council (mMRC) scale with a score $\geqslant 2$ signifying more breathlessness [21]. Chronic cough was defined as a cough lasting for $\geqslant 8$ weeks. Sputum production was defined as a duration of 3 months per year. HIV-related variables were extracted from patient records.

\section{Spirometry}

The procedure for spirometry has been previously described [17, 18]. An EasyOne spirometer (ndd Medical, Zürich, Switzerland) was used in accordance with American Thoracic Society/European Respiratory Society guidelines [22]. Predicted values and their lower limit of normal (LLN), i.e. the fifth percentile of the reference population, were calculated using multi-ethnic prediction equations provided by the Global Lung Function Initiative [23]. Airflow limitation was defined as either forced expiratory volume in $1 \mathrm{~s}(\mathrm{FEV} 1) /$ forced vital capacity (FVC) below the LLN or FEV1/FVC $<70 \%$ with $\mathrm{FEV}_{1}<80 \%$ predicted.

\section{CT scanning procedure}

PLWH and uninfected controls underwent a chest CT scan using the same two scanners at the same location (Department of Radiology, University Hospital of Copenhagen, Copenhagen, Denmark). Details regarding the scan protocol have previously been described [17, 24]. In brief, an Aquilion One Vision Edition scanner (Toshiba Medical Systems, Otawara-shi, Japan) was used for image acquisition with the following settings: $120 \mathrm{kVp}$, automated exposure control (SD15), and reconstruction with filtered back 
projection and a soft tissue kernel ( $1 \mathrm{~mm}$ slice thickness and $1 \mathrm{~mm}$ increment). Scans were acquired during a deep inspiratory breath-hold using spiral image acquisition including the entire lungs.

\section{Quantitative and visual emphysema assessment}

Lung emphysema was quantified by densitometry using \%LAA-950 [25-27], which is defined as the proportion of lung voxels of low density below a threshold of -950 Hounsfield Units (HU) and increases with worsening emphysema. Various cut-offs have been used to define emphysema [28, 29]. We primarily defined emphysema as \%LAA-950 $>10 \%$ but additionally used a threshold of $5 \%$. We also used the 15 th percentile density index (PD15) approach for lung density quantification [25]. PD15 is defined as the cut-off value in $\mathrm{HU}$ below which $15 \%$ of all voxels are distributed; it decreases with worsening emphysema. Images were scored using a dedicated lung density programme (Vitrea Vital Images, Minnetonka, MN, USA).

One board-certified radiologist (T.K.) scored all scans unblinded to HIV status but blinded to any other clinical information including results from quantitative CT analyses. A semi-quantitative score of $0-5$ was used $[4,30]$, where 0 indicates no emphysema (0\%); 1, trace emphysema (1-10\%); 2, mild emphysema (11-25\%); 3 , moderate emphysema (26-50\%); 4, severe emphysema (51-75\%); and 5, very severe (>75\%) visual emphysema.

\section{Statistical analysis}

Differences in clinical characteristics and emphysema outcomes were assessed using t-tests and MannWhitney comparisons for continuous data and Chi-squared tests or Fisher's exact test for categorical data. Logistic regression analyses were performed to determine risk factors associated with emphysema defined by \%LAA-950 according to a $5 \%$ and a $10 \%$ threshold. As a secondary outcome we assessed emphysema visually using a threshold of $\geqslant 1$ and $\geqslant 2$ [4]. Crude and adjusted odds ratios with $95 \%$ confidence intervals (CIs) were computed for these analyses. We additionally evaluated \%LAA-950 and PD15 as continuous outcomes and performed multiple linear regression analyses. For this purpose we log-transformed \%LAA-950 (due to a right skewed distribution) to approximate normality while the explanatory variables were kept in their original metrics. The interpretation for such a model is on the relative scale. For all regression analyses we considered models adjusted for age, sex, ethnicity and pack-years smoked and tested interactions between cumulative smoking and HIV status as well as the interaction between age and HIV status. We performed similar analyses using smoking status (defined as current, former, never or unknown) as a predictor, rather than pack-years, to avoid the potential colinearity between cumulative smoking and age. Finally, we evaluated PLWH separately and assessed whether various HIV-associated factors were each associated with emphysema. Statistical analyses were performed using $\mathrm{R}$ software version 3.3.2 [31].

\section{Results}

\section{Clinical characteristics}

A total of $742 \mathrm{PLWH}$ and 470 uninfected controls with quantitative and visual CT assessment were included in the study (table 1). The transmission modes among PLWH were primarily men having sex with men $(\mathrm{n}=517 ; 70.4 \%)$ and heterosexual $(\mathrm{n}=164 ; 22.3 \%)$. Most PLWH were receiving cART at the time of inclusion $(n=730 ; 98.6 \%)$ and had suppressed viral replication $(n=703 ; 95.4 \%)$. Few were intravenous drug users $(n=12 ; 1.6 \%)$ or co-infected with hepatitis $C(n=38 ; 5.1 \%)$. The current CD4 count in PLWH $\left(\right.$ mean \pm SD) was $712 \pm 288$ cells $\cdot \mathrm{mm}^{-3}$ and few had a CD4 count $<200$ cells $\cdot \mathrm{mm}^{-3}(\mathrm{n}=13 ; 1.8 \%)$. The nadir CD4 count was $235 \pm 178$ cells $\cdot \mathrm{mm}^{-3}$ with $346 \mathrm{PLWH}(47.5 \%)$ having a nadir CD4 count $<200$ cells $\cdot \mathrm{mm}^{-3}$.

\section{Quantitative and visual emphysema in PLWH and uninfected controls}

The prevalence of \%LAA-950 $>5 \%$ and $>10 \%$ were comparable between PLWH and uninfected controls (table 2). For PLWH, the prevalence of emphysema according to a threshold of $>5 \%$ was $21.2 \%$ (95\% CI 18.4-24.2) and of $>10 \%$ was $4.7 \%$ (95\% CI 3.4-6.5), and for uninfected controls was $24.3 \%$ (95\% CI $20.6-$ 28.3 ) and $4.0 \%$ (95\% CI 2.60-6.2). When evaluated as a continuous variable, \%LAA-950 appeared to be slightly lower in PLWH (2.0\%, 95\% CI 0.9-4.3 versus 2.7\%, 95\% CI 1.4-4.9; p<0.0001) (table 2, figure 1). Similar results were found for never-smokers (table 2). HIV was not associated with \%LAA-950 cut-offs for emphysema in multivariable logistic regression models adjusted for age, sex, ethnicity and pack-years of smoking (table 3). Moreover, we found no evidence for an interaction between smoking and HIV in these models ( $p$-interaction=0.82 and 0.74 ) or between age and HIV ( $p$-interaction=0.10 and 0.60 ). Likewise, in models adjusted for smoking status rather than cumulative smoking, we found no association between HIV and emphysema. We evaluated log-transformed \%LAA-950 as the outcome in a linear regression model and found HIV to be independently associated with a $29.0 \%$ (95\% CI 16.6-39.5) lower \%LAA-950 $(\mathrm{p}<0.0001)$. HIV was not independently associated with PD15 $\left(-1.94 \mathrm{~g} \cdot \mathrm{L}^{-1}, 95 \% \mathrm{CI}-4.6-0.75, \mathrm{p}=0.16\right)$. 


\begin{tabular}{|c|c|c|c|}
\hline & PLWH & Uninfected controls & $p$-value \\
\hline Subjects $n$ & 742 & 470 & \\
\hline Age & $54.2 \pm 9.2$ & $57.4 \pm 8.8$ & $<0.001$ \\
\hline Age groups years & & & $<0.001$ \\
\hline $40-50$ & 284 (38.3) & $107(22.8)$ & $<0.001$ \\
\hline$>50-60$ & $253(34.1)$ & $174(37.0)$ & \\
\hline$>60-70$ & 156 (21.0) & 164 (34.9) & \\
\hline$>70-80$ & $46(6.2)$ & $23(4.9)$ & \\
\hline$>80$ & $3(0.4)$ & $2(0.4)$ & \\
\hline Male & $636(85.7)$ & $386(82.1)$ & 0.11 \\
\hline Scandinavian & $552(75.6)$ & $429(92.1)$ & $<0.001$ \\
\hline BMI $\mathrm{kg} \cdot \mathrm{m}^{-2}$ & $24.9 \pm 3.7$ & $26.7 \pm 3.6$ & $<0.001$ \\
\hline Smoking status & & & $<0.001$ \\
\hline Current smokers & 195 (26.3) & $46(9.8)$ & \\
\hline Ex-smokers & $288(38.8)$ & 206 (43.8) & \\
\hline Never-smokers & 245 (33.0) & 217 (46.2) & \\
\hline $\begin{array}{l}\text { Tobacco consumption pack-years }{ }^{\#, ~} \text { median (IQR) } \\
\text { mMRC score }\end{array}$ & $19.5(7.4-33.0)$ & $18.0(7.5-32.0)$ & $\begin{array}{c}0.44 \\
<0.001\end{array}$ \\
\hline 0 & 480 (67.3) & 342 (72.9) & \\
\hline 1 & $177(24.8)$ & 114 (24.3) & \\
\hline 2 & $36(5.0)$ & $6(1.3)$ & \\
\hline 3 & $12(1.7)$ & $4(0.9)$ & \\
\hline 4 & $8(1.1)$ & $3(0.6)$ & \\
\hline \multicolumn{4}{|l|}{ Airflow limitation } \\
\hline $\mathrm{FEV}_{1} / \mathrm{FVC}<\mathrm{LLN}$ & $77(10.6)$ & $49(10.6)$ & 1.00 \\
\hline $\mathrm{FEV}_{1} / \mathrm{FVC}<0.7$ with $\mathrm{FEV}_{1}<80 \%^{+}$ & $69(9.5)$ & $36(7.8)$ & 0.37 \\
\hline FEV $1 \%$ predicted & & & $<0.001$ \\
\hline$>80$ & $567(78.0)$ & $408(88.1)$ & \\
\hline $50-79$ & $146(20.1)$ & 55 (11.9) & \\
\hline $30-49$ & $11(1.5)$ & $0(0)$ & \\
\hline$<30$ & $3(0.4)$ & $0(0)$ & \\
\hline Lung volume $e^{\delta} \mathrm{L}$ & $5.8 \pm 1.4$ & $5.6 \pm 1.8$ & 0.03 \\
\hline \multicolumn{4}{|c|}{ 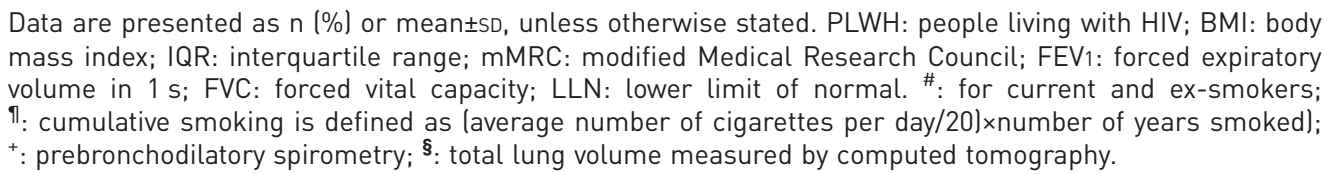 } \\
\hline
\end{tabular}

We also assessed emphysema visually. Individuals with visual emphysema were more likely to have a higher median \%LAA-950 than individuals without visual emphysema (5.0\%, 95\% CI 2.5-10.5 versus 2.2\%, 95\% CI 1.0-4.4; p<0.0001). In univariate analysis, HIV was associated with emphysema (OR 1.87, 95\% CI 1.11-3.27, p=0.02). However, we were not able to find this association in multivariate analyses adjusted for age, sex, ethnicity and cumulative smoking (adjusted OR 1.53, 95\% CI 0.85-2.83, p=0.16). We

TABLE 2 Quantification of emphysema in PLWH and uninfected controls

\begin{tabular}{|c|c|c|c|c|c|c|}
\hline & \multicolumn{3}{|c|}{ All individuals } & \multicolumn{3}{|c|}{ Never-smokers } \\
\hline & PLWH & Uninfected controls & p-value & PLWH & Uninfected controls & p-value \\
\hline Subjects n & 742 & 470 & & 245 & 217 & \\
\hline$\%$ LAA-950 > 10\% & $35(4.7)$ & $19(4.0)$ & 0.68 & $3(1.2)$ & $8(3.7)$ & 0.15 \\
\hline \%LAA-950 median (IQR) & $2.0(0.9-4.3)$ & $2.7(1.4-4.9)$ & $<0.0001$ & $1.8(0.8-3.9)$ & $2.5(1.5-4.6)$ & $<0.0001$ \\
\hline PD15 mean£sD & $109.6 \pm 24.8$ & $109.1 \pm 19.0$ & 0.72 & $112.7 \pm 25.8$ & $110.2 \pm 19.4$ & 0.25 \\
\hline
\end{tabular}

Data are presented as $\mathrm{n}(\%)$, unless otherwise stated. PLWH: people living with HIV; \%LAA-950: percentage of low attenuation area under 950 Hounsfield units; IQR: interquartile range; PD15: 15th percentile density index. 

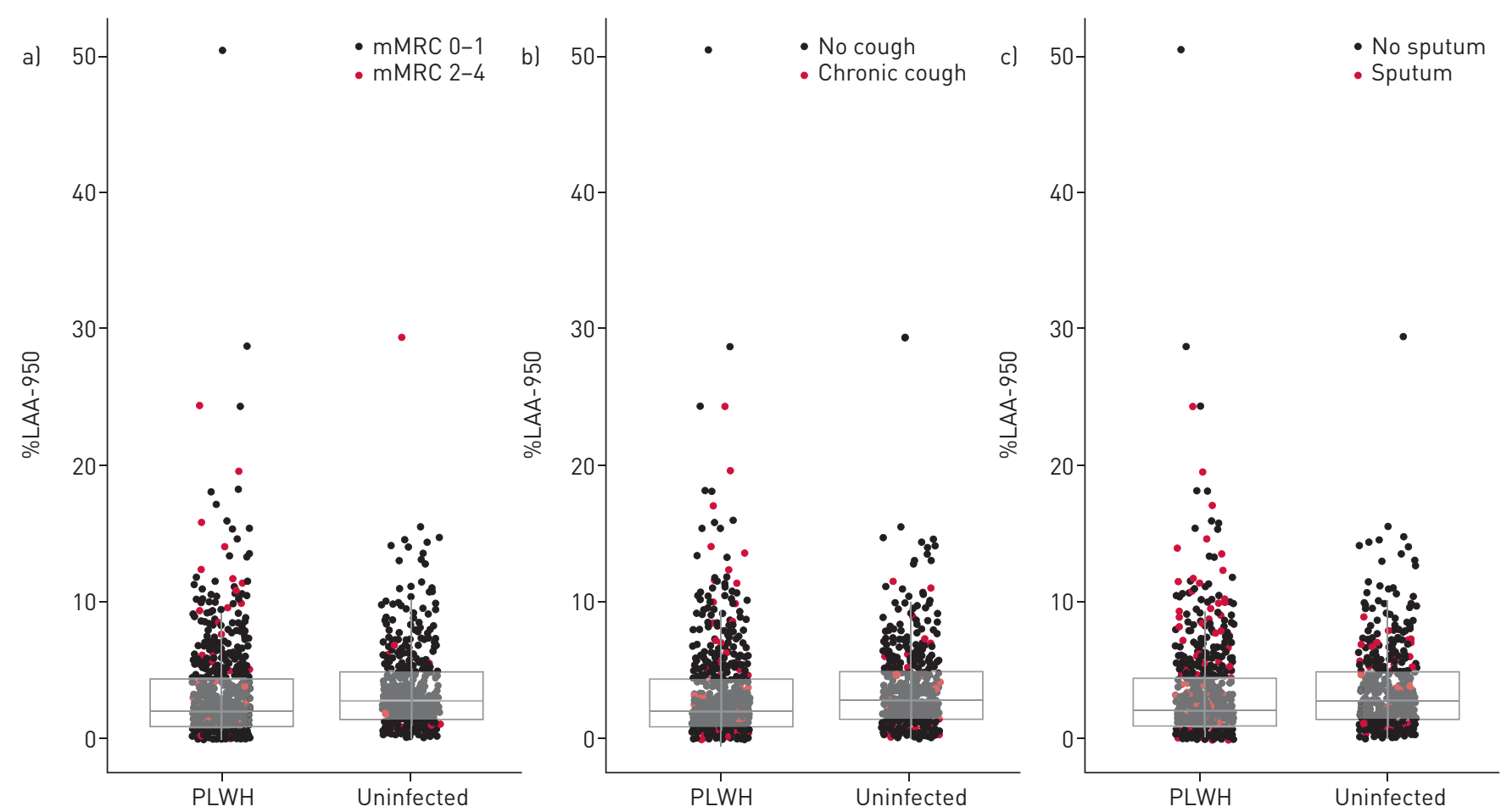

FIGURE 1 Percentage of low attenuation area under 950 Hounsfield units (\%LAA-950) and self-reported respiratory morbidity in people living with HIV (PLWH) and uninfected controls stratified by a) modified Medical Research Council dyspnoea scale (mMRC) score 0-1 or 2-4; b) no cough or chronic cough; and c) no sputum or sputum. $p$-value for the difference between \%LAA-950 in PLWH and uninfected controls (p<0.001). Boxplot represents 25 th and 75 th percentile (lower and upper quartiles, respectively) and 50th percentile (median).

found similar results when smoking exposure was modelled according to smoking status rather than cumulative smoking (adjusted OR 1.48, 95\% CI 0.83-2.73, p=0.19) and when visual emphysema was defined according to grade $1(1-10 \%)$ or higher (adjusted OR $0.88,95 \%$ CI $0.58-1.33, \mathrm{p}=0.54$ ).

\section{Associations with emphysema by HIV status}

We subsequently evaluated whether emphysema was more prevalent in PLWH with airflow limitation than in uninfected controls with airflow limitation. Emphysema (\%LAA-950 >10\%) tended to be more common in PLWH (17 out of 77) with FEV1/FVC <LLN than in controls (4 out of 49 ) with FEV1/FVC $<$ LLN ( $p=0.07$ ).

We also assessed the clinical significance of emphysema by evaluating the distribution of breathlessness (mMRC 2-4) in PLWH and uninfected controls with \%LAA-950 >10\%. Eight out of 35 PLWH with emphysema had breathlessness, and mMRC categories were different in PLWH with and without emphysema $(\mathrm{p}<0.001)$. In uninfected controls, one out of 19 individuals had breathlessness and mMRC categories did not differ according to emphysema $(\mathrm{p}=0.18)$ (figure 1). Sputum production was present in 12 out of 35 PLWH with \%LAA-950 $>10 \%$ and more common than in individuals without emphysema $(\mathrm{p}<0.01)$. Sputum was present in zero out of 19 uninfected controls with emphysema and, thus, not more

TABLE 3 Multivariate logistic regression analyses to determine whether HIV is independently associated with \%LAA-950

\begin{tabular}{|c|c|c|c|c|c|c|c|c|}
\hline & \multicolumn{4}{|c|}{$\%$ LAA-950 >5\% } & \multicolumn{4}{|c|}{$\%$ LAA-950 >10\% } \\
\hline & OR (95\% CI) & p-value & $\mathrm{aOR}^{\#}(95 \% \mathrm{Cl})$ & p-value & OR $(95 \% \mathrm{CI})$ & p-value & $\mathrm{aOR}^{\#}(95 \% \mathrm{Cl})$ & p-value \\
\hline HIV yes versus no & $0.83(0.64-1.11)$ & 0.21 & $0.96(0.71-1.29)$ & 0.77 & $1.18(0.67-2.12)$ & 0.58 & $1.25(0.68-2.36)$ & 0.48 \\
\hline $\begin{array}{l}\text { Pack-years of smoking } \\
\text { per } 10 \text { pack-years }\end{array}$ & $1.06(0.99-1.14)$ & 0.09 & $1.00(0.92-1.08)$ & 0.99 & $1.23(1.09-1.39)$ & $<0.001$ & $1.15(1.01-1.31)$ & 0.03 \\
\hline
\end{tabular}

\%LAA-950: percentage of low attenuation area under 950 Hounsfield units; aOR: adjusted odds ratio. ${ }^{\#}$ : multivariate model adjusted for age, sex, ethnicity, pack-years of smoking and HIV. 
TABLE 4 Multivariate logistic regression analyses to determine whether HIV-related factors are independently associated with $\%$ LAA-950

\begin{tabular}{|c|c|c|c|c|c|c|c|c|}
\hline & \multicolumn{4}{|c|}{$\%$ LAA-950 >5\% } & \multicolumn{4}{|c|}{$\%$ LAA-950 >10\% } \\
\hline & OR $(95 \% \mathrm{CI})$ & p-value & $\mathrm{aOR}^{\#}(95 \% \mathrm{CI})$ & $p$-value & OR $(95 \% \mathrm{CI})$ & p-value & $\mathrm{aOR}^{\#}(95 \% \mathrm{Cl})$ & p-value \\
\hline Current $\mathrm{CD} 4$ per 100 cells $\cdot \mathrm{mm}^{-3}$ & $0.97(0.91-1.03)$ & 0.38 & $0.99(0.93-1.07)$ & 0.98 & $1.06(0.95-1.18)$ & 0.28 & $1.08(0.96-1.21)$ & 0.16 \\
\hline HIV viral load $>50$ copies $\cdot \mathrm{mL}^{-1}$ & $0.96(0.38-2.12)$ & 0.92 & $0.84(0.29-2.07)$ & 0.72 & $2.03(0.47-6.08)$ & 0.26 & $2.43(0.53-8.07)$ & 0.19 \\
\hline Previous PCP yes versus no & $1.26(0.65-2.33)$ & 0.47 & $0.85(0.39-1.71)$ & 0.66 & $1.63(0.47-4.31)$ & 0.38 & $1.49(0.42-4.14)$ & 0.49 \\
\hline Time living with HIV per 5 years & $1.18(1.07-1.31)$ & $<0.01$ & $1.09(0.97-1.22)$ & 0.15 & $1.24(1.02-1.51)$ & 0.03 & $1.06(0.86-1.33)$ & 0.57 \\
\hline
\end{tabular}

\%LAA-950: percentage of low attenuation area under 950 Hounsfield units; aOR: adjusted odds ratio; PCP: pneumocystis pneumonia.

\#: multivariate model adjusted for age, sex, ethnicity, pack-years of smoking and HIV.

common in those without emphysema $(\mathrm{p}=0.24)$. Chronic cough was not significantly associated with emphysema in PLWH $(\mathrm{p}=0.07)$ or uninfected controls $(\mathrm{p}=0.65)$ (figure 1).

\section{HIV-related factors and emphysema}

Current CD4 count, nadir CD4 count, detectable viral replication and prior pneumocystis pneumonia were not associated with either the 5\% or 10\% cut-off for \%LAA-950 after adjusting for age, sex, ethnicity and pack-years smoked (table 4). Time living with HIV (per 5 years) was associated with \%LAA-950 in univariate analyses but not after multivariate adjustments (table 5). Although a CD4 nadir $<200$ cells $\cdot \mathrm{mm}^{-3}$ appeared to be associated with visual emphysema (OR 1.70, 95\% CI 0.97-3.02, p<0.07), this association was not found in multivariate analyses (table 5). None of the other HIV variables were associated with visual emphysema in uni- or multivariate analyses.

\section{Discussion}

Our findings contrast with pre-ART studies, and a limited number of cART-era studies evaluating emphysema visually, which found HIV to be independently associated with emphysema. HIV was not independently associated with emphysema and we found no evidence of an interaction between age and tobacco smoking with HIV status. We also found no association between factors associated with HIV disease and emphysema. However, emphysema seemed to be more common in PLWH with airflow limitation than in uninfected controls with airflow limitation, and breathlessness was more strongly associated with emphysema in PLWH than in uninfected controls.

In previous studies radiographic emphysema in PLWH has been semi-quantitatively determined by visual assessment [3, 4, 13, 16]. Emphysema was highly prevalent in these studies, ranging from $15 \%$ [3] to as high as $31 \%$ [16]. However, concordance between visual and quantitative CT assessment for the presence or absence of emphysema is only moderate [28]. Each modality has certain strengths and limitations. On the one hand, visual scoring has the advantage that the radiologist can distinguish whether the low attenuation is caused by emphysematous destruction or hyper-inflated regions consistent with gas-trapping caused by small airways disease [32]. Moreover, current smoking status per se may be associated with higher lung density (possibly due to inflammation and an accumulation of soot and tar) [33], which could theoretically lower the extent of emphysema, i.e. a lower \%LAA-950, in PLWH. On the other hand, quantitative measurements correlate better with microscopic and macroscopic emphysema than visual scoring [34], may be less prone to over-estimating the extent of emphysema [32] and provide better reproducibility [35]. Few quantitative CT studies have been performed in PLWH [14, 15, 36, 37]. The largest of these studies was the Lung-HIV study from three US centres performing quantitative emphysema determination in 510 PLWH [14]. Although this study included more current smokers, a higher percentage of intravenous drug users and a higher number of individuals with detectable viral replication than the present study, it found $29.4 \%$ with emphysema according to the $>5 \%$ \%AA-950 threshold and $5.7 \%$ according to the $>10 \%$ threshold. Despite the fact that our study consisted of individuals $>40$ years, $\sim 6$ years older on average, we found comparable prevalence of emphysema $(21.2 \%$ and $4.7 \%)$.

Few studies have included uninfected controls. One study compared mean lung density, but not specific \%LAA-950 thresholds, in 83 PLWH and 42 uninfected controls, and found HIV to be independently associated with higher lung density [37]. These findings may suggest other underlying disease processes, e.g. inflammation and fibrosis, but did not indicate an association between HIV status and emphysema. HIV was found to be a risk factor for semi-quantitative emphysema $(>10 \%)$ in the Veteran Aging Cohort 
Study (VACS) sub-study of 114 PLWH and 89 uninfected controls [4]. We were not able to reproduce this finding in any of our models using quantitative CT measures, and we found no evidence of an interaction between HIV status and age or HIV status and cumulative smoking. Thus, although we did not study lung density trajectories, the effects of age and smoking seemed to be equally important in PLWH and uninfected controls, in contrast to what has previously been suggested [3]. Although earlier findings and the present results differ, they may both be correct and reflect a changing disease pattern for lung diseases in PLWH as a result of better and earlier therapy. Our cohort is unique in that included individuals from a high-resource setting with a near complete cART coverage, few intravenous drug users and individuals with rather high CD4 counts. Thus, other mediators of HIV, such as viral replication, inflammation or immune deficiency in the absence of cART, may potentially be responsible for the development of emphysematous destruction. We did not find an association between emphysema (quantitative or visual) and CD4 nadir. However, the VACS sub-study found a CD4 nadir $<200$ cells $\cdot \mathrm{mm}^{-3}$ to be an independent risk factor for emphysema (OR 2.98, 95\% CI 1.14-7.81) in a model adjusted for cumulative smoking [4]. Although the VACS cohort tended to have poorer immune status and this association may have been confounded by other factors, we have previously found that the CD4 nadir is associated with measures of pulmonary function in the present cohort [18]. Moreover, a longitudinal study using VACS registry data showed an association between time-updated CD4 counts and lung cancer [38]. Thus, exposure of low CD4 counts before treatment initiation may render the host more susceptible to smoking and other potential insults contributing to emphysema. The discrepancy between our study and the VACS data may reflect the inclusion of different study populations, e.g. the VACS cohort included a large proportion of individuals who still smoked $(\sim 60 \%)$ and had used intravenous drugs $(\sim 30 \%)$ or marijuana $(\sim 85 \%)$. As a consequence, the effects of other risk factors, such as CD4 nadir, may differ.

A few studies assessing overlap between emphysema and airflow limitation have suggested that emphysema may occur independently of airflow limitation $[15,39]$. We found emphysema to be more prevalent in PLWH with airflow limitation $\left(\mathrm{FEV}_{1} / \mathrm{FVC}<\mathrm{LLN}\right)$ than in uninfected controls with airflow limitation. Also, FEV1 \% predicted correlated better with emphysema in PLWH than in uninfected controls. We previously determined $\mathrm{FEV} 1 / \mathrm{FVC}<\mathrm{LLN}$ to be equally prevalent in PLWH and uninfected controls, but the degree of airflow limitation was more severe in PLWH [18]. These findings suggest that the emphysematous phenotype is more prevalent in PLWH with airflow limitation and contributes to a more severe degree of airflow limitation.

We also observed that breathlessness and sputum production were more common in PLWH with emphysema than in uninfected controls with emphysema. Similarly, a VACS sub-study found that emphysema only correlated with objective functional impairment (assessed by the 6-min walking distance) and self-reported chronic cough in PLWH and not uninfected controls [39]. Taken together, these findings suggest that the clinical significance of emphysema may differ according to HIV status, although the mechanism for this differential impact is unknown.

Our study has limitations and strengths. First, uninfected controls were not completely matched on demographic variables and risk factors: tobacco smoking was more common in PLWH and the two populations had different ethnicities. Despite accounting for these factors, using several different lung density measures (\%LAA-950 according to 5\% and 10\% thresholds, PD15 and log-transformed \%LAA-950) and visual assessment, we were not able to show that HIV is associated with emphysema. Second, visual assessment of CT was unblinded to HIV status but was nevertheless consistent with results from the quantitative measures. Finally, we were not able to evaluate the exposure to low CD4 counts longitudinally and had to rely on static measures.

In conclusion, HIV was not independently associated with emphysema in a well-resourced setting where most PLWH had undetectable viral replication and high CD4 counts. Moreover, we were not able to find evidence that the effect of smoking is different according to HIV status. However, the clinical significance of emphysema was greater in PLWH, and emphysema seemed to be more prevalent in PLWH with airflow limitation than in uninfected individuals with airflow limitation.

Acknowledgments: We thank all the study subjects for their participation. We thank the staff at the Dept of Infectious Diseases at Rigshospitalet and at Hvidovre Hospital for their dedicated participation. The support of Per Sievertsen and Andreas Fuchs, the Cardiac CT-Copenhagen General Population SubStudy, Rigshospitalet is highly appreciated.

Author contributions: A. Ronit was responsible for the concept, data collection and statistical analysis, and drafted the manuscript. T. Kristensen was responsible for the concept and data collection, and edited the manuscript. V.S. Hoseth was responsible for data collection and edited the manuscript. D. Abou-Kassem was responsible for data collection and edited the manuscript. J.T. Kühl was responsible for the concept and edited the manuscript. T. Benfield was responsible for the concept and edited the manuscript. J. Gerstoft was responsible for the concept and edited the 
manuscript. S. Afzal was responsible for the concept and data collection, and edited the manuscript. B.G. Nordestgaard was responsible for the concept and data collection, and edited the manuscript. J.D. Lundgren was responsible for the concept and edited the manuscript. J. Vestbo was responsible for the concept and edited the manuscript. K.F. Kofoed was responsible for the concept and edited the manuscript. S.D. Nielsen was responsible for the concept and data collection, and edited the manuscript.

Conflict of interest: T. Benfield reports personal fees for advisory board activities from Bristol Myers Squibb, Gilead and Abbvie, personal fees for lectures including service on speakers bureau from Bristol Myers Squibb and GSK, personal fees for development of educational presentations from Bristol Myers Squibb and GSK, and non-financial support (travel/accommodation/meeting expenses) from Bristol Myers Squibb and Gilead, outside the submitted work. J. Gerstoft reports honoraria for consulting and presenting paid to his institution from Gilead, Abbvie, ViiV, BMS, MSD, Janssen and Medivir, outside the submitted work. J. Vestbo reports honoraria for consulting and presenting from AstraZeneca, Boehringer Ingelheim, Chiesi, GSK and Novartis, outside the submitted work. K.F. Kofoed reports personal fees for advisory board activity from Gilead, outside the submitted work. S.D. Nielsen reports unrestricted research grants from Novo Nordisk Foundation, Lundbeck Foundation, Augustinus Foundation and Rigshospitalet Research Council, travelling grants from Gilead, MSD, BMS and GSK/ViiV, and advisory board activity for Gilead and GSK/ViiV, outside the submitted work.

Support statement: This work was supported by Rigshospitalet Research Council, Region Hovedstaden, The Lundbeck Foundation, The Novo Nordisk Foundation, The Danish National Research Foundation grant 126 and AP Møller og hustru Chastine McKinney Møllers Fond. The study was designed, conducted, analysed and written by the authors without involvement of any commercial party. Funding information for this article has been deposited with the Crossref Funder Registry.

\section{References}

1 Kuhlman JE, Knowles MC, Fishman EK, et al. Premature bullous pulmonary damage in AIDS: CT diagnosis. Radiology 1989; 173: 23-26.

2 Diaz PT, Clanton TL, Pacht ER. Emphysema-like pulmonary disease associated with human immunodeficiency virus infection. Ann Intern Med 1992; 116: 124-128.

3 Diaz PT, King MA, Pacht ER, et al. Increased susceptibility to pulmonary emphysema among HIV-seropositive smokers. Ann Intern Med 2000; 132: 369-372.

4 Attia EF, Akgun KM, Wongtrakool C, et al. Increased risk of radiographic emphysema in HIV is associated with elevated soluble CD14 and nadir CD4. Chest 2014; 146: 1543-1553.

5 Twigg HL, Soliman DM, Day RB, et al. Lymphocytic alveolitis, bronchoalveolar lavage viral load, and outcome in human immunodeficiency virus infection. Am J Respir Crit Care Med 1999; 159: 1439-1444.

6 Cribbs SK, Guidot DM, Martin GS, et al. Anti-retroviral therapy is associated with decreased alveolar glutathione levels even in healthy HIV-infected individuals. PLoS One 2014; 9: e88630.

7 Diaz PT, Wewers MD, King M, et al. Regional differences in emphysema scores and BAL glutathione levels in HIV-infected individuals. Chest 2004; 126: 1439-1442.

8 Cribbs SK, Lennox J, Caliendo AM, et al. Healthy HIV-1-infected individuals on highly active antiretroviral therapy harbor HIV-1 in their alveolar macrophages. AIDS Res Hum Retroviruses 2015; 31: 64-70.

9 Costiniuk CT, Jenabian MA. The lungs as anatomical reservoirs of HIV infection. Rev Med Virol 2014; 24: 35-54.

10 Kaner RJ, Santiago F, Crystal RG. Up-regulation of alveolar macrophage matrix metalloproteinases in HIV1(+) smokers with early emphysema. J Leukoc Biol 2009; 86: 913-922.

11 Chung NPY, Ou X, Khan KMF, et al. HIV reprograms human airway basal stem/progenitor cells to acquire a tissue-destructive phenotype. Cell Rep 2017; 19: 1091-1100.

12 Ronit A, Mathiesen IH, Gelpi M, et al. Small airway dysfunction in well-treated never-smoking HIV-infected individuals. Eur Respir J 2017; 49: 1602186.

13 Guaraldi G, Besutti G, Scaglioni R, et al. The burden of image based emphysema and bronchiolitis in HIV-infected individuals on antiretroviral therapy. PLoS One 2014; 9: e109027.

14 Leader JK, Crothers K, Huang L, et al. Risk factors associated with quantitative evidence of lung emphysema and fibrosis in an HIV-infected cohort. J Acquir Immune Defic Syndr 2016; 71: 420-427.

15 Samperiz G, Guerrero D, Lopez M, et al. Prevalence of and risk factors for pulmonary abnormalities in HIV-infected patients treated with antiretroviral therapy. HIV Med 2014; 15: 321-329.

16 Triplette M, Attia EF, Akgun KM, et al. A low peripheral blood CD4/CD8 ratio is associated with pulmonary emphysema in HIV. PLoS One 2017; 12: e0170857.

17 Ronit A, Haissman J, Kirkegaard-Klitbo DM, et al. Copenhagen comorbidity in HIV infection (COCOMO) study: a study protocol for a longitudinal, non-interventional assessment of non-AIDS comorbidity in HIV infection in Denmark. BMC Infect Dis 2016; 16: 713.

18 Ronit A, Lundgren J, Afzal S, et al. Copenhagen Co-morbidity in HIV Infection (COCOMO) study group. Airflow limitation in people living with HIV and matched uninfected controls. Thorax 2018; 73: 431-438.

19 Colak Y, Afzal S, Nordestgaard BG, et al. Majority of never-smokers with airflow limitation do not have asthma: the Copenhagen General Population Study. Thorax 2016; 71: 614-623.

20 Kobylecki CJ, Vedel-Krogh S, Afzal S, et al. Plasma urate, lung function and chronic obstructive pulmonary disease: a Mendelian randomisation study in 114979 individuals from the general population. Thorax 2017; in press [https://doi.org/10.1136/thoraxinl-2017-210273].

21 Vogelmeier CF, Criner GJ, Martinez FJ, et al. Global Strategy for the Diagnosis, Management, and Prevention of Chronic Obstructive Lung Disease 2017 Report: GOLD Executive Summary. Eur Respir J 2017; 49: 1750214.

22 Miller MR, Hankinson J, Brusasco V, et al. Standardisation of spirometry. Eur Respir J 2005; 26: 319-338.

23 Quanjer PH, Stanojevic S, Cole TJ, et al. Multi-ethnic reference values for spirometry for the 3-95-yr age range: the global lung function 2012 equations. Eur Respir J 2012; 40: 1324-1343.

24 Ronit A, Kristensen T, Klitbo DM, et al. Incidental lung cancers and positive computed tomography images in people living with HIV. AIDS 2017; 31: 1973-1977. 
25 Coxson HO, Dirksen A, Edwards LD, et al. The presence and progression of emphysema in COPD as determined by CT scanning and biomarker expression: a prospective analysis from the ECLIPSE study. Lancet Respir Med 2013; 1: 129-136.

26 Lynch DA, Al-Qaisi MA. Quantitative computed tomography in chronic obstructive pulmonary disease. J Thorac Imaging 2013; 28: 284-290.

27 Woodruff PG, Barr RG, Bleecker E, et al. Clinical significance of symptoms in smokers with preserved pulmonary function. N Engl J Med 2016; 374: 1811-1821.

28 Barr RG, Berkowitz EA, Bigazzi F, et al. A combined pulmonary-radiology workshop for visual evaluation of COPD: study design, chest CT findings and concordance with quantitative evaluation. COPD 2012; 9: 151-159.

29 Johannessen A, Skorge TD, Bottai M, et al. Mortality by level of emphysema and airway wall thickness. Am J Respir Crit Care Med 2013; 187: 602-608.

30 Wilson DO, Weissfeld JL, Balkan A, et al. Association of radiographic emphysema and airflow obstruction with lung cancer. Am J Respir Crit Care Med 2008; 178: 738-744.

31 R Development Core Team. R: a language and environment for statistical computing. Vienna, Austria, $\mathrm{R}$ Foundation for Statistical Computing, 2008.

32 Gietema HA, Muller NL, Fauerbach PV, et al. Quantifying the extent of emphysema: factors associated with radiologists' estimations and quantitative indices of emphysema severity using the ECLIPSE cohort. Acad Radiol 2011; 18: 661-671.

33 Ashraf $\mathrm{H}$, Lo P, Shaker SB, et al. Short-term effect of changes in smoking behaviour on emphysema quantification by CT. Thorax 2011; 66: 55-60.

34 Bankier AA, De M V, Keyzer C, et al. Pulmonary emphysema: subjective visual grading versus objective quantification with macroscopic morphometry and thin-section CT densitometry. Radiology 1999; 211: 851-858.

35 Cavigli E, Camiciottoli G, Diciotti S, et al. Whole-lung densitometry versus visual assessment of emphysema. Eur Radiol 2009; 19: 1686-1692.

36 Clausen E, Wittman C, Gingo M, et al. Chest computed tomography findings in HIV-infected individuals in the era of antiretroviral therapy. PLoS One 2014; 9: e112237.

37 Drummond MB, Lambert AA, Hussien AF, et al. HIV infection is independently associated with increased CT scan lung density. Acad Radiol 2017; 24: 137-145.

38 Sigel K, Wisnivesky J, Crothers K, et al. Immunological and infectious risk factors for lung cancer in US veterans with HIV: a longitudinal cohort study. Lancet HIV 2017; 4: e67-e73.

39 Triplette M, Attia E, Akgun K, et al. The differential impact of emphysema on respiratory symptoms and 6-minute walk distance in HIV infection. J Acquir Immune Defic Syndr 2017; 74: e23-e29. 